FTUAM 96-7

21-nov-1996

\title{
Pomerons and Jet Events at HERA
}

\author{
J. Vermaseren \\ NIKHEF \\ P.O.Box 41882 \\ NL-1009DB, Amsterdam \\ F. Barreiro, L. Labarga and F. J. Ynduráin \\ Departamento de Física Teórica, C-XI, \\ Universidad Autónoma de Madrid, \\ Canto Blanco, \\ E-28034 Madrid, SPAIN.
}

\begin{abstract}
We study two and three jet events with a large rapidity gap at HERA. Unlike in the IngelmanSchlein approach we do not adscribe a structure to the Pomeron. Instead, the coupling of the Pomeron to quarks or gluons is taken pointlike, which makes the model easy to test: the only degrees of freedom are the coupling constants of the Pomeron to the quarks or the gluons and a cutoff procedure to keep the Pomeron-gluon coupling well behaved.
\end{abstract}




\section{Introduction}

The prediction of the Pomeron as a pseudo particle has inspired a large number of experiments in the 60's and 70's. Although effective parameters could be determined under the assumption that the particle exists, its existence was never shown unambiguously. Recently the so-called 'large rapidity gap' events at HERA [1] have caused new excitement about the Pomeron hypothesis. These events show jet activity far away from the beam pipe, but there is no trace of a color string between these jets and the remnants of the proton. Actually it is not excluded that in many or even most cases the proton remains intact. Hence the events carry the signature of a diffractive mechanism in which a color neutral object reacts with the virtual photon that comes from the electron. The outcoming events can be classified by the number of jets they show. This is similar to $e^{-} e^{+} \rightarrow$ hadrons with which these events can be shown to have much in common. We will comment on this in the results section.

Thus far most theoretical analysis has concerned the two jet events. Various models have been tried, ranging from a full calculation of a gluon pair exchange [2] and [3], to a phenomenological description of Pomeron structure functions [4] [5] and even to a gluon photon fusion scheme in which the resulting quark pair somehow sheds its color and leaves it behind inside the proton fragments $[$ [ $]$. In this paper we will produce the Pomeron with a classically parameterized diffractive mechanism [7]. Having produced it, we will couple it to quarks and/or gluons with a pointlike coupling. This has been tried before for two jet events, but then in the case of a small $Q^{2}$ of the photon [8]; see also ref [9]. In this paper we are however interested in the region of large $Q^{2}$ in which the outgoing electron gets observed clearly inside the detector. In addition we will have a look at three jet events. The aim of the paper is then to see how well the events can be used to determine the coupling of the Pomeron to the quarks (and/or gluons).

There are various motivations for this model. First it is the simplest model one can think of, and hence it is necessary to have at least a look at it. Another motivation has to do with the fact that the model contains explicit calculations for three-jet production allowing us to make simple predictions for broadening effects in the multihadronic diffractive final states. In addition the matrix elements can be implemented in a Monte Carlo programme using the Lund fragmentation scheme. Thus, our model can be directly compared to experimental data. In fact, as it will be shown in a forthcoming publication [10], the model gives a good description of the data.

\section{The computation}

Considering the fact that we insist on seeing the electron, we can treat it in the normal way. This means that it is just another particle in the trace calculations, and we do not have to take extra precautions with respect to numerical stability of either the matrix element or the kinematics. Because of this there is absolutely no need to use an equivalent photon approximation. If the electron were to stay in the beampipe the situation would be different. In the forward direction terms of order $m_{e}^{2} / s$ are important and seemingly leading terms cancel each other to a high degree of accuracy. This not being the case we can work out the trace of the Feynman diagrams by brute force using the program FORM [11] and evaluate the resulting FORTRAN code without much worry. The two jet diagrams of figure 1 are easy to compute. 


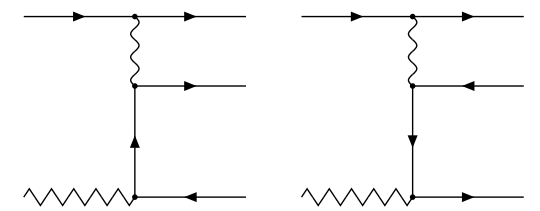

Figure 1: The 2 diagrams for Pomeron induced two jet production. The Pomeron is denoted by the zigzag line at the left bottom of each diagram

In this case there is only one 'new' parameter. The strength of the coupling of the Pomeron to the quarks: $g_{P q q}$. Basically the normalization of the reaction fixes this constant. The cross section is easily calculated. If one were to ignore the polarization of the photon and the 'mass' of the Pomeron the matrix element of the subprocess

$$
\gamma(q)+\mathrm{P}(\mathrm{p}) \rightarrow \bar{q}\left(k_{1}\right)+q\left(k_{2}\right)
$$

(in brackets the momenta of the particles) then its cross section, denoted by $\hat{\sigma}_{\gamma \mathrm{P}}$ would be

$$
d \hat{\sigma}_{\gamma \mathrm{P}}=\frac{2 g_{P q q}^{2} \alpha}{\pi} \frac{s-q^{2}}{u t} \delta\left(p+q-k_{1}-k_{2}\right) \frac{d^{3} k_{1}}{2 k_{10}} \frac{d^{3} k_{2}}{2 k_{20}} .
$$

Here $t, u$ are the standard Mandelstam variables $\left(t\right.$ is defined by $\mathrm{P}$ and $\left.k_{1}\right)$, and $g_{P q q}$ is defined by the effective interaction Lagrangian $\mathcal{L}_{\text {eff }}=g_{P q q} \bar{q} q P$. In practice we did not ignore the polarization of the photon and the 'mass' of the Pomeron. Hence the formula for the full process is a little longer:

$$
\begin{aligned}
d \sigma_{e P}= & \frac{d^{3} k_{1}}{2 E_{1}} \frac{d^{3} k_{2}}{2 E_{2}} \frac{d^{3} p_{e}^{\text {out }}}{2 E_{e}^{\text {out }}} \delta^{4}\left(p_{e}+P-k_{1}-k_{2}-p_{e}^{\text {out }}\right)\left(-q^{2} \frac{\left(M_{X}^{2}-q^{2}\right)^{2}+P^{2}}{u t}+P^{2}\left(q^{2}\right)^{2}\left(\frac{1}{u^{2}}+\frac{1}{t^{2}}\right)\right. \\
& +8 P \cdot p_{e}\left(\frac{1}{u}+\frac{1}{t}\right)\left(\frac{p_{e} \cdot k_{1}\left(P^{2}-u\right)}{t}+\frac{p_{e} \cdot k_{2}\left(P^{2}-t\right)}{u}\right) \\
& \left.-8\left(P \cdot p_{e}\right)^{2} \frac{M_{X}^{2}}{u t}-8 P^{2} p_{e} \cdot k_{1} p_{e} \cdot k_{2}\left(\frac{1}{u}+\frac{1}{t}\right)^{2}\right) \frac{g_{P q q}^{2} \alpha^{2}}{\left(q^{2}\right)^{2} \pi^{3}\left(s-P^{2}\right)}
\end{aligned}
$$

We use here $M_{X}$ for the diquark invariant mass, $p_{e}$ for the incoming electron and $p_{e}^{\text {out }}$ for the outgoing electron.

The three parton diagrams, responsible mostly for the three jet events, are given in figure 2 . Here the formulae are much more complicated, but with the use of FORM we obtain an expression of about 800 terms which can be written into a file in such a way that it can be included into a FORTRAN program directly. We have now another 'new' parameter: $g_{P g g}$, the coupling of the Pomeron to two gluons. Its size can be fixed by looking at the ratio between two and three jet events. It should be noted that because the Pomeron is taken to be a scalar particle and because we assumed that the quarks are massless, the six quark-coupling induced diagrams do not interfere with the two gluon-coupling induced diagrams. 

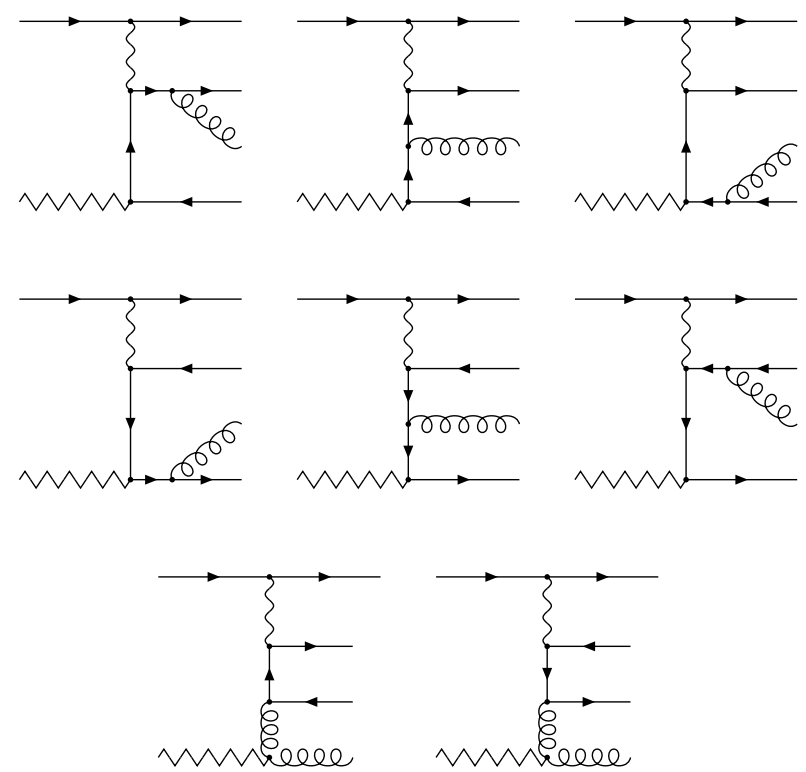

Figure 2: The 6+2 diagrams for Pomeron induced three jet production

When the Pomeron is coupled to two gluons we use a $g_{P g g} F^{\mu \nu} F_{\mu \nu}$ coupling. This presents some problems. Because this coupling causes an unphysical high energy behaviour, due to the extra dimension, this is 'repaired' by a form factor. For this form factor we took the expression $1 /\left(M^{2}-t_{3}\right)^{1 / 2}$ in which $t_{3}$ is the invariant mass of the internal gluon. It has a negative value. $M^{2}$ is a free parameter which is expected to be of the order of $1 \mathrm{GeV}^{2}$. Hence we used this value. The presence of the formfactor is noticeable in the differential cross section with respect to $M_{X}$ which is the invariant mass of the three jet system. In the case that we do not use the form factor we get a clearly unphysical behaviour for large values of $M_{X}$. It is roughly constant until phase space cuts it off. With the formfactor a more physical behaviour is obtained and the mass distribution looks similar to the corresponding one for the 6 diagrams with the quark coupling.

For the production of the Pomeron we used a phenomenological fit that has been obtained by Donnachie and Landshoff [7]. This way the Pomeron flux in a proton can be written as:

$$
f_{P}\left(x_{P}, t\right)=\frac{9 \beta_{0}^{2}}{4 \pi^{2}}\left(F_{1}(t)\right)^{2} x_{P}^{-2 \alpha(t)+1}
$$

with

$$
\begin{aligned}
F_{1}(t) & =\frac{4 m_{p}^{2}-A t}{4 m_{p}^{2}-t}\left(1-\frac{t}{B}\right)^{-2} \\
A & =2.8 \\
B & =0.7 \\
\beta_{0} & =3.24 \mathrm{GeV}^{-2} \\
\alpha(t) & =1+\epsilon+\alpha^{\prime} t \\
\epsilon & =0.085 \\
\alpha^{\prime} & =0.25
\end{aligned}
$$

It is of course rather easy to implement such a flux factor, provided that the integrations over phase space are done numerically. It is equally possible to change the intercept $\epsilon$ to a value close to 0.2 as suggested by recent HERA data. 
Next we need to do the integration. This is done by means of a Monte Carlo program VEGAS [12]. It is a so-called self learning program that integrates over a unit cube of a given number of dimensions. Hence we have to transform points in such a cube into points in phase space. The routines that do this are called the kinematics routines. In the current calculation we use some 'standard' two and three body decay routines. Both routines assume that the 'decay' takes place in the CM frame of the decaying object. This means that at various points we need some trivial Lorenz transformations. The two body decay routine is used for the reaction $e^{-} P \rightarrow e^{-} X$ in which $P$ is the Pomeron and $X$ represents the 2 or 3 jet system. It is also used for the subreaction $\gamma^{*} P \rightarrow 2$ jets. The three body decay routine first selects two Dalitz variables and then supplements them with the proper CM angles. It is used for the subreaction $\gamma^{*} P \rightarrow 3$ jets. Because everything happens at large angles such routines are sufficient.

Of course integration would be meaningless if we would not apply acceptance cuts. One has to worry about two sets of cuts. In the first set we try, at the parton level, to imitate the experimental analysis. In this case we do as if the partons are the jets of the experiment and we copy the analysis cuts of the experiment. In the second case we prepare the program for event generation allowing the experimental analysis programs to apply fragmentation into hadrons. We can then do the analysis at this level. Alternatively the resulting particles can be sent to the detector emulator programs. This last step would be part of a full experimental analysis. In the case of hadronization the cuts have to be stricter, because the fragmentation will make things less clear. There will be events that pass the analysis cuts this way that did not pass them in the theoretical analysis, et vice versa. In general the last method is superior, but it requires the application of the full experimental detector simulators. It also requires many more computer resources. The first method however is usually sufficient to give a good idea of what is happening, how distributions will differ between various models, etc. The study of the hadronization effects, without using the detector emulators, gives already a good indication of whether things will change much in a full analysis. The cuts we use in the calculations at the parton level are:

- $\operatorname{Rapitity}(\eta)$ of the scattered electron: $>-3.7$.

- Energy of the scattered electron: $>5 \mathrm{GeV}$.

- $Q^{2}$ of the electron: $>3 \mathrm{GeV}^{2}$.

- Mass of the outcoming hadron $\operatorname{system}\left(M_{X}\right)$ : $>3 \mathrm{GeV}$.

- Angle of the hadron system: $\cos \theta<0.9$.

- Rapidity of the individual partons: $<3$.

- Transverse energy of the individual partons: $>0.2 \mathrm{GeV}$.

- $M_{j j}^{2} / M_{X}^{2} \geq Y_{\min }=0.05$.

- Beam pipe cut: Each parton has $\theta>0.1 \mathrm{rad}$.

For the calculation that involves the hadronization we used

- For the jets apply the cuts for the individual partons.

- Only particles with $\theta>0.1 \mathrm{rad}$ are used.

- There should be at least 4 particles. 
- All particles should have a rapidity $<2.5$.

- Angle of the hadron system: $\cos \theta<0.75$.

- $Q^{2}$ of the electron: $>10 \mathrm{GeV}^{2}$.

- Mass of the outcoming hadron $\operatorname{system}\left(M_{X}\right)$ : $>5 \mathrm{GeV}$.

The hadronization was performed with the program JETSET 7.4 [13]. The parton flavors were selected to be d:u:s = 1:4:1 and the decays of some of the hadrons (mainly the strange ones) were suppressed.

Because we use a Monte Carlo integration method, it is easy to make a large variety of histograms. In addition we can use an additional set of programs after the integration that makes the program behave like an event generator in which the events all have weight one. Hence the programs are suitable for a detailed experimental analysis. The number for the total cross section can usually be obtained with an accuracy of a few tenth of a percent during the integration phase.

\section{Results}

In diffractive $e-p$ events, the absence of the proton remnant considerably simplifies the analysis of the multihadronic final state. If interpreted in terms of $\gamma^{*}-P$ collisions, the Pomeron fractional momentum $\xi$, with respect to the proton beam, can be easily obtained from the relation

$$
\xi=\frac{M_{X}^{2}+Q^{2}}{M_{X}^{2}+W^{2}}
$$

where $M_{X}^{2}, Q^{2}$ and $W^{2}$ stand for the usual DIS kinematic variables.

We propose to establish an analogy between $\gamma^{*}-P$ scattering at a centre of mass given by $M_{X}$ and $e^{+} e^{-}$annihilation at a centre of mass energy $\sqrt{s}$. In this section, we study the dependence of shape variables describing the multihadronic final states as a function of $M_{X}$, in order to asses the importance of the two different broadening mechanisms in the model

gluon radiation

a) Pomeron splitting into two gluons

To $O\left(\alpha_{s}\right)$, the cross-sections for the three types of processes considered in this paper, within the cuts described in the previous section and for a fixed value of the strong coupling constant $\alpha_{s}=0.25$, are:

- $(43.83 \pm 0.08) \cdot g_{P q q}^{2} n b$ for two-jet production due to the direct coupling of the Pomeron to quark pairs

- $(3.240 \pm 0.003) \cdot g_{P q q}^{2} n b$ for three-jet production from gluon bremstrahlung to the procees above

- $(0.2705 \pm 0.0009) \cdot g_{P g g}^{2} n b$ for three-jet production from the Pomeron coupling to gluon pairs

In Fig. 3a we show $\frac{1}{\sigma} \frac{d \sigma}{d M_{X}}$ for these three reactions. Notice that three-jet processes through the Pomeron coupling to gluons tend to give rise to larger masses then those coming from bremstrahlung. In Fig. 3b we show the thrust [14] distribution for three parton final states in the $\gamma-P$ centre of mass frame. Again, the tail in the thrust distribution associated with final states due to 
Pomeron coupling to two gluons, dashed line, is harder than that associated to bremstrahlung from the Pomeron to two quarks coupling, solid line. In fact, the latter is in rather good agreement with the analytical expression derived by Ellis, Gaillard and Ross for $e^{+} e^{-}$annihilation [15].
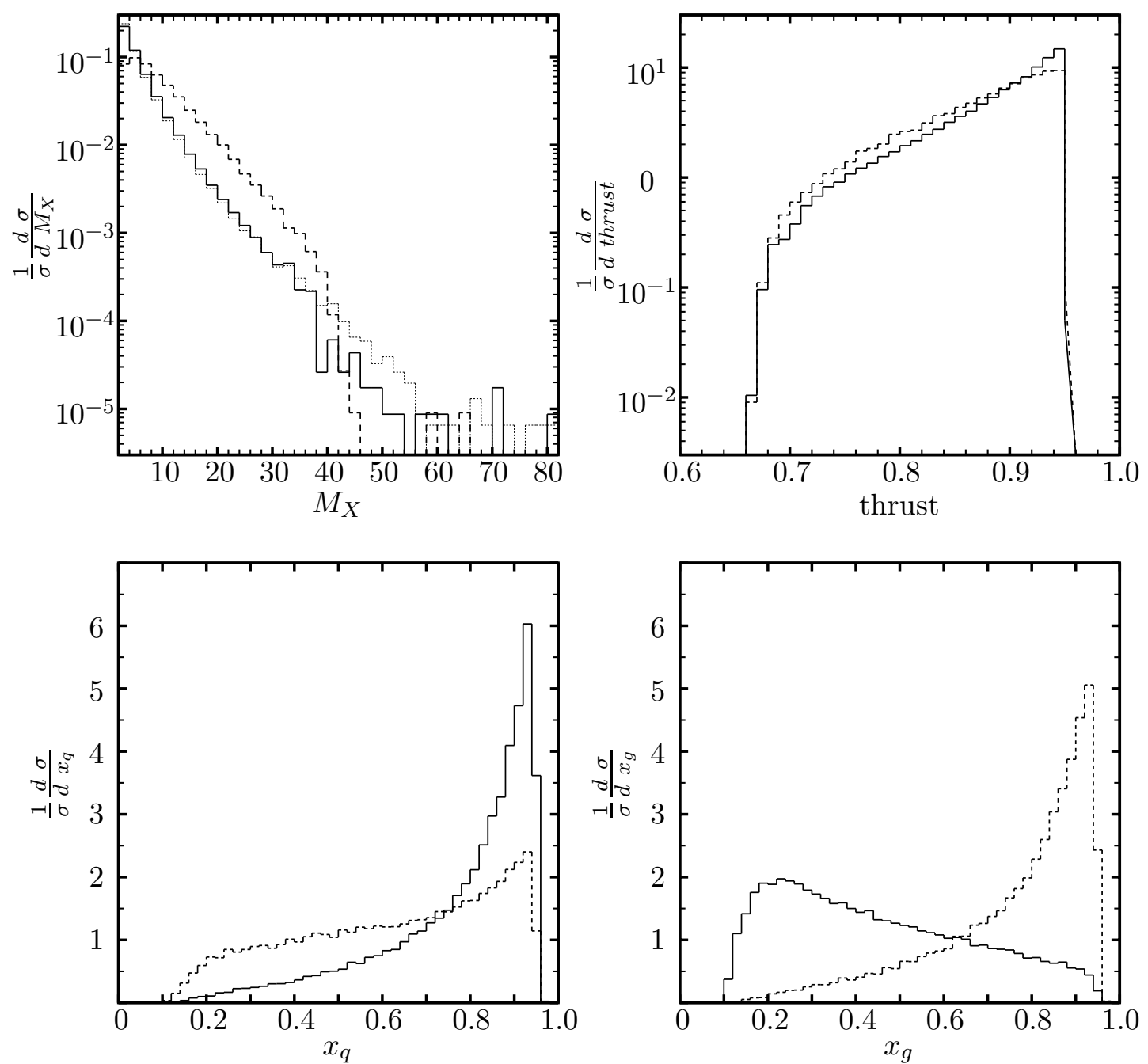

Figure 3: The distributions at the parton level.

The solid line refers to the production of three partons, using the $P q q$ vertex. The dashed line corresponds to the Pgg vertex. The dotted line in the figure at the top left refers to the production of two partons.

To deepen our insight into the roles played by gluons in the two different three-parton processes we are considering, it is instructive to look at the parton fractional momenta, defined as usual by $x_{q}=2 E_{q} / \Sigma E_{h a d r}$ and $x_{g}=2 E_{g} / \Sigma E_{h a d r}$. They are presented in Figures 3c and 3d respectively. It is striking to see that while bremstrahlung gluons are soft partons as expected, those coming from Pomeron splitting are much harder.

In order to study to what extent the properties exhibited above are masked by fragmentation effects, we have calculated the thrust distributions in the hadronic centre of mass, after fragmentation, for various ranges in $M_{X}$. 

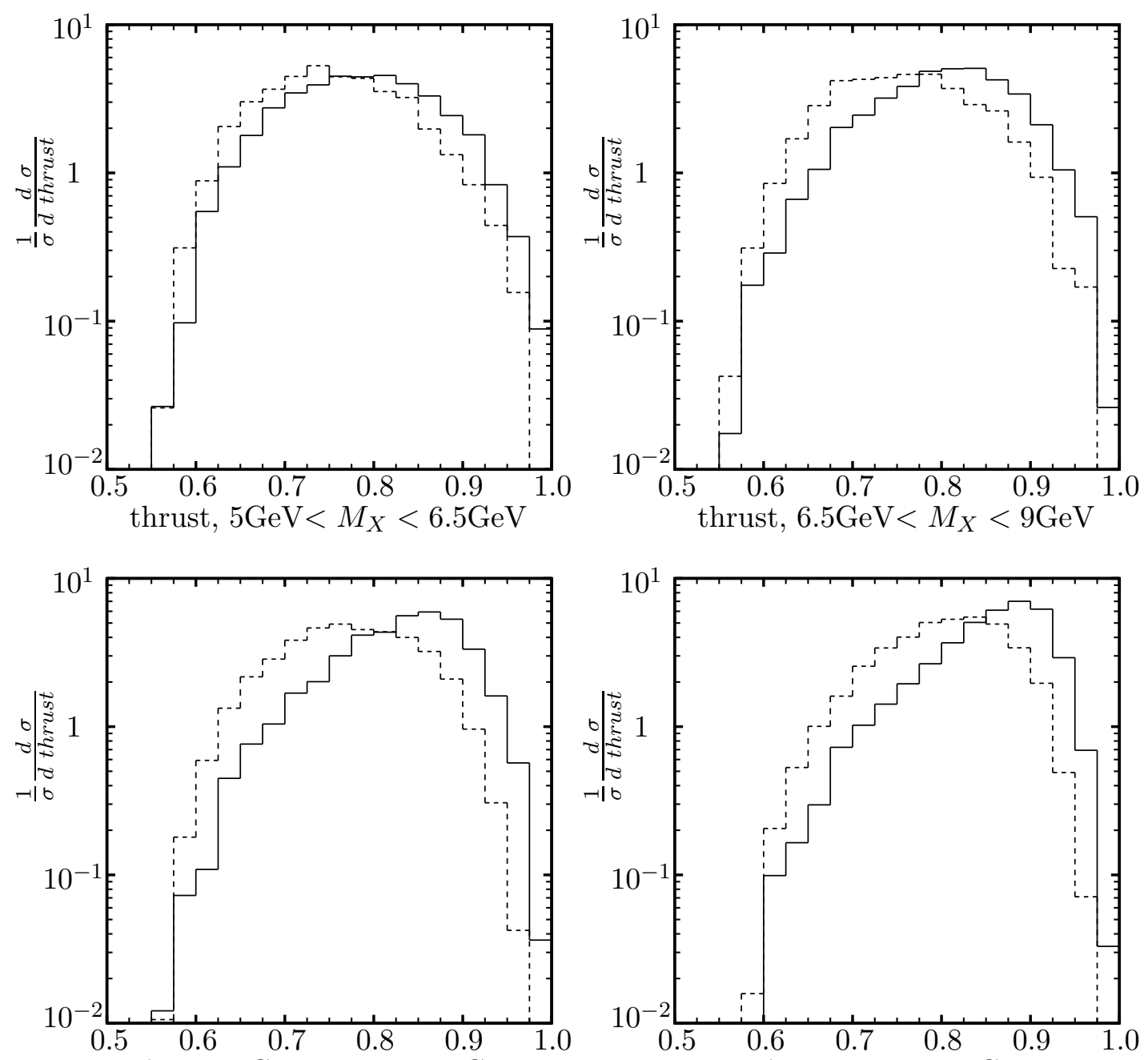

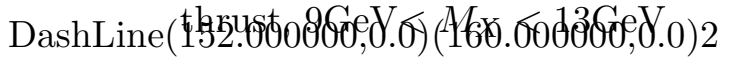

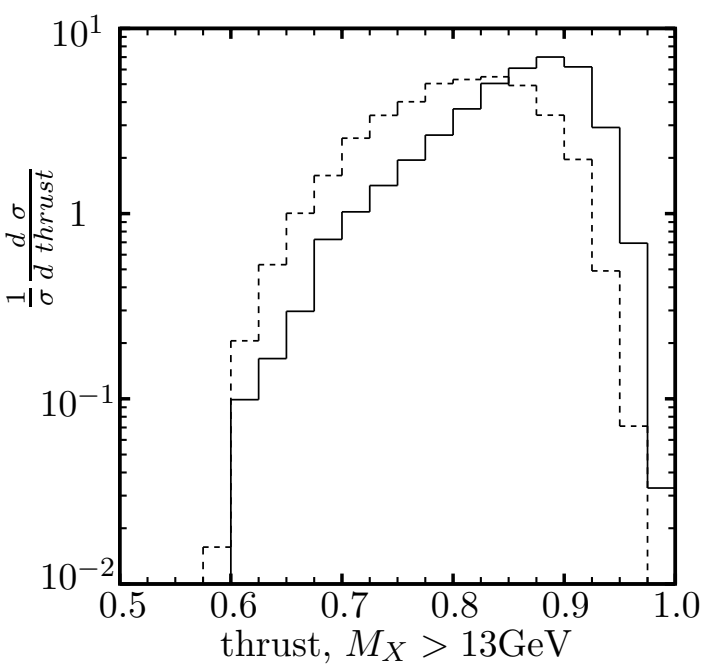

Figure 4: The thrust distributions at the hadron level.

The solid line refers to the production of three partons, using the $P q q$ vertex. The dashed line corresponds to the Pgg vertex.

The results are shown in Fig. 4. Notice that while at low masses, both thrust distributions, i.e. the one stemming from the $P \rightarrow q \bar{q}$ coupling and that due to $P \rightarrow g g$ are similar, at high masses, the latter gives rise to multihadronic final states which are more spherical than the former.

To summarize and conclude. We have calculated the cross-sections to $O\left(\alpha_{s}\right)$ for diffractive $e-p$ scattering mediated by Pomeron emission. We have considered a direct coupling of the Pomeron to quark and gluon pairs. If the Pomeron couples preferentially to gluons as recently indicated by experimental data 16, the multihadronic final states should exhibit, as the hadronic masses become large, broadening effects much stronger than those associated to gluon bremstrahlung in $e^{+} e^{-}$annihilation [17].

One of the authors (J.V.) likes to thank the Universidad Autónoma in Madrid for its kind hospitality.

\section{References}


[1] ZEUS Coll., M. Derrick et al., Phys. Lett. B315 (1993) 481.

[2] A. Donnachie and P.V. Landshoff, Phys. Lett. B285 (1992) 172.

[3] N.N. Nikolaev and B.G. Zakharov, Z.Phys. C53 (1992) 331.

[4] G. Ingelman and P. Schlein, Phys. Lett. B152 (1985) 256 This model has been implemented in a Monte Carlo programme, POMPYT, by P. Bruni and G. Ingelman

[5] H. Jung, Comp. Phys. Comm. 86 (1995) 147.

[6] W. Buchmüller and A. Hebecker, Phys. Lett. B355 (1995) 573

[7] A. Donnachie and P.V. Landshoff, Phys. Lett. 191B (1987) 309, Nucl.Phys. B303 (1988) 634.

[8] B.A. Kniehl, H.G. Kohrs and G. Kramer, DESY 94-140

[9] J. Bartels, H. Lotter and M. Wusthoff Phys. Lett. B379 (1996)239. erratum-ibid. B382 (1996) 449.

[10] The ZEUS collaboration, to be published.

[11] J.A.M. Vermaseren, Symbolic Manipulation with FORM, published by CAN (Computer Algebra Nederland), Kruislaan 413, 1098 SJ Amsterdam, 1991, ISBN 90-74116-01-9. An earlier version of FORM can be obtained for free by means of anonymous ftp from ftp.nikhef.nl.

[12] G.P. Lepage, J. Comp. Phys. 27 (1978) 192

[13] T. Sjostrand, Computer Physics Commun. 82 (1994) 74. The most recent long description (unpublished) is T. Sjostrand, CERN-TH.7112/93 (1993, revised August 1994).

[14] E. Fahri, Phys. Rev. Lett. 39 (1977) 1436 and S. Brandt et al., Phys. Lett. 12 (1964) 57

[15] J. Ellis, M.K. Gaillard and G.G. Ross, Nucl. Phys. B111 (1976) 253

[16] ZEUS Coll., M. Derrick et al., Phys. Lett. B356 (1995) 129

[17] TASSO Coll., R. Brandelik et al, Phys. Lett. 86B (1979) 243, MARK J Coll, D.P. Barber et al., Phys. Rev. Lett. 43 (1979) 830, PLUTO Coll. Ch. Berger et al., Phys. Lett. B86B (1979) 418 JADE Coll., W. Bartel et al., Phys. Lett. B 91 (1980) 142 